\title{
LINEAR ISOMORPHISMS PRESERVING GREEN'S RELATIONS FOR MATRICES OVER ANTI-NEGATIVE SEMIFIELDS
}

DOI:

10.1016/j.laa.2018.01.023

\section{Document Version}

Accepted author manuscript

Link to publication record in Manchester Research Explorer

\section{Citation for published version (APA):}

Guterman, A., Johnson, M., \& Kambites, M. (2018). LINEAR ISOMORPHISMS PRESERVING GREEN'S RELATIONS FOR MATRICES OVER ANTI-NEGATIVE SEMIFIELDS. Linear Algebra and its Applications. https://doi.org/10.1016/j.laa.2018.01.023

\section{Published in:}

Linear Algebra and its Applications

\section{Citing this paper}

Please note that where the full-text provided on Manchester Research Explorer is the Author Accepted Manuscript or Proof version this may differ from the final Published version. If citing, it is advised that you check and use the publisher's definitive version.

\section{General rights}

Copyright and moral rights for the publications made accessible in the Research Explorer are retained by the authors and/or other copyright owners and it is a condition of accessing publications that users recognise and abide by the legal requirements associated with these rights.

\section{Takedown policy}

If you believe that this document breaches copyright please refer to the University of Manchester's Takedown Procedures [http://man.ac.uk/04Y6Bo] or contact uml.scholarlycommunications@manchester.ac.uk providing relevant details, so we can investigate your claim.

\section{OPEN ACCESS}




\title{
LINEAR ISOMORPHISMS PRESERVING GREEN'S RELATIONS FOR MATRICES OVER ANTI-NEGATIVE SEMIFIELDS
}

\author{
ALEXANDER GUTERMAN ${ }^{1}$, MARIANNE JOHNSON ${ }^{2}$ \\ and MARK KAMBITES ${ }^{3}$
}

\begin{abstract}
In this paper we characterize those linear bijective maps on the monoid of all $n \times n$ square matrices over an anti-negative semifield (that is, a semifield which is not a field) which preserve each of Green's equivalence relations $\mathcal{L}, \mathcal{R}, \mathcal{H}, \mathcal{D}, J$ and the corresponding four preorderings $\leq_{\mathcal{L}}, \leq_{\mathcal{R}}, \leq_{\mathcal{H}}, \leq_{J}$. These results apply in particular to the tropical and boolean semirings.
\end{abstract}

Key words: Green's relations, linear preservers, semifield, tropical semiring, boolean semiring.

Mathematics Subject Classification : 15A86 (primary); 15A80, 16Y60, 12K10 (secondary).

\section{INTRODUCTION}

Given an equivalence or order relation on a certain algebraic system, it is natural to ask: what are the transformations that can be performed on this system that leave the relation invariant? In the case where the algebraic system under consideration is a matrix algebra over a field, the investigation of such transformations dates back to the following result of Frobenius [5], which gives a characterisation of the bijective complex linear transformations for which the determinant is an invariant:

Theorem 1.1. (Frobenius 1897) Let $\mathbb{C}$ be the field of complex numbers, and let $T: M_{n}(\mathbb{C}) \rightarrow M_{n}(\mathbb{C})$ be a bijective linear transformation such that $\operatorname{det} T(X)=\operatorname{det} X$ for all matrices $X \in M_{n}(\mathbb{C})$. Then there exist invertible matrices $U, V \in M_{n}(\mathbb{C})$, with $\operatorname{det}(U V)=1$, such that either $T(X)=U X V$ for all matrices $X \in M_{n}(\mathbb{C})$, or $T(X)=U X^{T} V$ for all $X \in M_{n}(\mathbb{C})$, where $X^{T}$ denotes the transposed matrix.

This result was subsequently generalized by Schur [15], who gave a characterisation of maps preserving all subdeterminants of any fixed order $r$. Later Dieudonné [4] proposed a new approach to classify such transformations, based on the fundamental theorem of projective geometry. Dieudonné

\footnotetext{
${ }^{1}$ Faculty of Algebra, Department of Mathematics and Mechanics, Moscow State University, GSP-1, 119991 Moscow, Russia.

${ }^{2}$ School of Mathematics, University of Manchester, Manchester M13 9PL, UK. Email Marianne. Johnson@maths . manchester . ac.uk.

${ }^{3}$ School of Mathematics, University of Manchester, Manchester M13 9PL, UK. Email Mark. Kambites@manchester . ac .uk.
} 
obtained a standard characterization of bijective linear maps preserving the set of singular matrices over arbitrary fields.

Following on from these initial investigations, many authors have studied the problem of determining the linear operators on the $n \times n$ matrix algebra $M_{n}(F)$ over a field $F$ that leave certain matrix relations, subsets, or properties invariant (see the surveys $[11,12]$ for the details). In the last two decades much attention has been paid to the investigation of maps preserving different invariants for matrices over various semirings, where completely different techniques are necessary to obtain a classification of linear transformations with certain preserving properties; see [12, Section 9.1] and references therein for more details.

In this paper we consider Green's relations for the full monoids of $n \times n$ matrices over anti-negative semifields (that is, semifields which are not themselves fields), including most notably the tropical and boolean semirings. Green's relation are five equivalence relations $(\mathcal{L}, \mathcal{R}, \mathcal{H}, \mathcal{D}$ and $\mathcal{J})$ and four pre-orders $\left(\leq_{\mathcal{R}}, \leq_{\mathcal{L}}, \leq_{\mathcal{H}}\right.$ and $\left.\leq_{J}\right)$ which can be defined on any semigroup. They encapsulate the divisibility relations between elements and hence together describe the structure of the maximal subgroups and principal left, right, and two-sided ideals of the semigroup. Green's relations play an important role in many aspects of semigroup theory. A natural problem is to characterize all bijective linear maps which preserve each of the orders or equivalence relations defined by Green. In Section 2 we briefly recall the necessary definitions, pointing out that a semifield is either a field, or else an anti-negative semifield, and that in the latter case (or more generally for any anti-negative semiring without zero divisors [3, Theorem 2.14]) the bijective linear maps have a very restricted form. For an anti-negative semifield $\mathcal{S}$, we then give a complete characterisation of those bijections which preserve each of the relations $\mathcal{L}, \mathcal{R}, \mathcal{D}, \mathcal{J}$ and the pre-orders $\leq_{\mathcal{R}}, \leq_{\mathcal{L}}$ and $\leq_{\mathcal{J}}$ on $M_{n}(S)$ in Section 3, and characterise the bijections which preserve the relation $\mathcal{H}$ and the pre-order $\leq_{\mathcal{H}}$ in $M_{n}(\mathcal{S})$ in Section 4.

\section{GREen's RELATIONS AND FACTOR RANK IN MATRIX SEMigROUPS}

Let $\mathcal{M}$ be a monoid. For $a, b \in \mathcal{M}$ we say that:

(i) $a \leq_{\mathcal{R}} b$ if and only if $a \mathcal{M} \subseteq b \mathcal{M}$, that is, if there exists $s \in \mathcal{M}$ with $a=b s$. We say that $a \mathcal{R} b$ if $a \leq_{\mathcal{R}} b$ and $b \leq_{\mathcal{R}} a$, or in other words if $a$ and $b$ generate the same principal right ideal of $\mathcal{M}$.

(ii) $a \leq_{\mathcal{L}} b$ if and only if $\mathcal{M} a \subseteq \mathcal{M} b$, that is, if there exists $s \in \mathcal{M}$ with $a=s b$. We say that $a \mathcal{L} b$ if $a \leq_{\mathcal{L}} b$ and $b \leq_{\mathcal{L}} a$, or in other words if $a$ and $b$ generate the same principal left ideal of $\mathcal{M}$.

(iii) $a \leq_{\mathcal{J}} b$ if and only if $\mathcal{M} a \mathcal{M} \subseteq \mathcal{M} b \mathcal{M}$, that is, if there exist $s, t \in \mathcal{M}$ with $a=s b t$. We say that $a \mathcal{J} b$ if $a \leq_{\mathcal{J}} b$ and $b \leq_{\mathcal{J}} a$, or in other words, if $a$ and $b$ generate the same principal two-sided ideal of $\mathcal{M}$.

(iv) $a \mathcal{D} b$ if and only if there exists $c \in \mathcal{M}$ such that $a \mathcal{R} c$ and $c \mathcal{L} b$ (or equivalently, if there there exists $c \in \mathcal{M}$ such that $a \mathcal{L} c$ and $c \mathcal{R} b)$.

(v) $a \mathcal{H} b$ if and only if $a \mathcal{R} b$ and $a \mathcal{L} b$.

(vi) $a \leq_{\mathcal{H}} b$ if and only if $a \leq_{\mathcal{R}} b$ and $a \leq_{\mathcal{L}} b$.

We recall that a semiring is a set $\mathcal{S}$ together with two binary operations, addition and multiplication, such that $\mathcal{S}$ is a commutative monoid under 
addition (with identity denoted by 0 ); $\mathcal{S}$ is a semigroup under multiplication (with identity, if any, denoted by 1 ); multiplication is distributive over addition on both sides; and $s 0=0 s=0$ for all $s \in \mathcal{S}$. The semiring $\mathcal{S}$ is said to be commutative if the multiplication is commutative. It is said to be anti-negative if $a+b=0$ implies that both $a$ and $b$ are equal to 0 . It is a semifield if $\mathcal{S} \backslash\{0\}$ is an abelian group under multiplication. We begin with a few simple observations concerning semifields.

Remark 2.1. It is easily verified that a semifield is either anti-negative or a field; we give a short proof of this fact, with no particular claim of originality. Let $\mathcal{S}$ be a semifield and let $a, b \in \mathcal{S}$ with $a+b=0$. Clearly, if one of $a$ or $b$ is 0 , then the other must be as well. Suppose then that neither $a$ nor $b$ is 0 , so that $\mathcal{S}$ is not anti-negative. Since $\mathcal{S}$ is a semifield, the non-zero element $a$ has an inverse $a^{-1} \in \mathcal{S} \backslash\{0\}$. Multiplying $a+b=0$ through by $a^{-1}$ then yields $1+a^{-1} b=0$. But this provides an additive inverse to 1 , and hence to every element $x \in \mathcal{S}$ since $0=x\left(1+a^{-1} b\right)=x+x a^{-1} b$. Thus $\mathcal{S}$ is a field.

Remark 2.2. If $\mathcal{S}=\{0,1\}$ is a 2-element semifield then it is easy to see that the axioms determine the values of all sums and products except for $1+1$. Setting $1+1=0$ yields the field $\mathbb{Z}_{2}$, while setting $1+1=1$ yields the boolean semiring, which we shall denote by $\mathbb{B}$. Since $\mathbb{Z}_{2}$ is a field and the definition of a semifield implies $0 \neq 1$, we see that $\mathbb{B}$ is the unique anti-negative semifield with fewer than three elements. Some of our results about anti-negative semifields will be established by one argument requiring the ability to take three distinct elements, and a separate argument for the special case of $\mathbb{B}$.

Remark 2.3. The multiplicative group of any semifield which is not a field is torsion-free (see [14, Lemma 1.2] for a short proof, for example). In particular, every anti-negative semifield $\mathcal{S}$ except for $\mathbb{B}$ contains an invertible element $k$ such that $k^{2} \neq 1$.

Remark 2.4. A semiring $\mathcal{S}$ is called idempotent if $a+a=a$ for all $a \in \mathcal{S}$. An idempotent semiring is necessarily anti-negative; indeed if $a+b=0$ then $0=a+b=a+a+b=a+0=a$ and a dual argument gives $0=b$. For example, the tropical semifield $\mathbb{R}_{\max }:=\mathbb{R} \cup\{-\infty\}$ together with addition given by taking the maximum and multiplication given by extending addition of real numbers so as to make $-\infty$ a zero element, is an idempotent and hence anti-negative semifield. (Note that the real number 0 is the multiplicative identity here.)

Let $\mathcal{S}$ be a semiring. We write $\mathcal{S}^{i \times j}$ for the set of $i \times j$ matrices over $\mathcal{S}$, which forms an $\mathcal{S}$-module in the obvious way. We write $M_{n}(\mathcal{S})$ for $\mathcal{S}^{n \times n}$ viewed as a semigroup under the matrix multiplication induced by the operations in $\mathcal{S}$. If $\mathcal{S}$ contains a multiplicative identity element 1, then $M_{n}(\mathcal{S})$ is a monoid, with obvious identity element.

Given an anti-negative semifield, our aim is to characterise those bijective $\mathcal{S}$-linear maps

$$
T: M_{n}(\mathcal{S}) \rightarrow M_{n}(\mathcal{S}),
$$

which preserve each of the pre-orders $\leq_{\mathcal{R}}, \leq_{\mathcal{L}}, \leq_{\mathcal{H}}, \leq_{\mathcal{J}}$ and each of the relations $\mathcal{R}, \mathcal{L}, \mathcal{J}, \mathcal{D}, \mathcal{H}$. We say that the map $T$ preserves the relation $\mathcal{P}$ if 
$A \mathcal{P} B$ implies that $T(A) \mathcal{P} T(B)$ for all $A, B \in M_{n}(\mathcal{S})$. We say that map $T$ strongly preserves $\mathcal{P}$ if for all $A, B \in M_{n}(\mathcal{S})$ we have $A \mathcal{P} B$ if and only if $T(A) \mathcal{P} T(B)$. For example, it is straightforward to verify that any transformation of the form $T(A)=P A Q$, where $P$ and $Q$ are invertible matrices over $\mathcal{S}$ will strongly preserve each of the described relations. The transposition transformation strongly preserves $\leq \mathcal{J}, \mathcal{J}, \mathcal{D}$ and $\mathcal{H}$ but it does not preserve any of the other relations (the $\mathcal{L}$ and $\mathcal{R}$ relations being transposed).

We begin by recalling a number of module-theoretic characterisations of Green's relations for matrix semigroups. For $A \in M_{n}(\mathcal{S})$ we write $\operatorname{Row}_{\mathcal{S}}(A)$ to denote the left $\mathcal{S}$-submodule of $\mathcal{S}^{1 \times n}$ generated by the rows of $A$ and $\operatorname{Col}_{\mathcal{S}}(A)$ to denote the right $\mathcal{S}$-submodule of $\mathcal{S}^{n \times 1}$ generated by the columns of $A$. The following statement is well-known and can be found in the literature for example as [7, Proposition 4.1].

Theorem 2.5. Let $\mathcal{S}$ be a semiring with multiplicative identity element, and $n \in \mathbb{N}$. For $A, B \in M_{n}(\mathcal{S})$ we have

(i) $A \leq_{\mathcal{L}} B$ if and only if $\operatorname{Row}_{\mathcal{S}}(A) \subseteq \operatorname{Row}_{\mathcal{S}}(B)$;

(ii) $A \leq_{\mathcal{R}} B$ if and only if $\operatorname{Col}_{\mathcal{S}}(A) \subseteq \operatorname{Col}_{\mathcal{S}}(B)$;

(iii) $A \mathcal{L} B$ if and only if $\operatorname{Row}_{\mathcal{S}}(A)=\operatorname{Row}_{\mathcal{S}}(B)$;

(iv) $A \mathcal{R} B$ if and only if $\operatorname{Col}_{\mathcal{S}}(A)=\operatorname{Col}_{\mathcal{S}}(B)$;

(v) $A \mathcal{H} B$ if and only if $\operatorname{Row}_{\mathcal{S}}(A)=\operatorname{Row}_{\mathcal{S}}(B)$ and $\operatorname{Col}_{\mathcal{S}}(A)=\operatorname{Col}_{\mathcal{S}}(B)$.

For semirings (or even semifields) in general, the $\mathcal{D}$ and $\mathcal{J}$ relations are harder to characterise in terms of row and column spaces. However, characterisations are known for many important semirings including in particular the tropical semiring $\mathbb{R}_{\max }$ (see $[1,7,9,17]$ ).

Recall that the factor rank $\operatorname{rk}(A)$ of a matrix $A \in \mathcal{S}^{n \times m}$ is the smallest positive integer $k$ such that $A=B C$ for some $B \in \mathcal{S}^{n \times k}$ and $C \in \mathcal{S}^{k \times m}$. By convention, a matrix with only zero entries has factor rank 0. Factor rank is also known as Schein rank (especially over the Boolean semiring) or Barvinok rank (especially over the tropical semiring).

Remark 2.6. Let $\mathcal{S}$ be a semifield and suppose that $M \in M_{n}(\mathcal{S})$ is a matrix with exactly four non-zero entries which are arranged in a square submatrix as follows:

$$
\left(\begin{array}{ll}
a & b \\
c & d
\end{array}\right)
$$

Then it is easy to see that $M$ has factor rank at most 2 and, moreover, $M$ has factor rank 2 if and only if $a d \neq b c$.

Remark 2.7. Factor rank respects the $\mathcal{J}$-order, in the sense that $A \leq \mathcal{J} B$ implies $\operatorname{rk}(A) \leq \operatorname{rk}(B)$ (see for example [9, Corollary 8.3]). It follows that it is an invariant of $\mathcal{H}-, \mathcal{L}$-, $\mathcal{R}-, \mathcal{D}$ - and $\mathcal{J}$-classes. Note that in general $A \leq_{\mathcal{R}} B$ and $\operatorname{rk}(A)=\operatorname{rk}(B)$ do not suffice to conclude that $A \mathcal{R} B$. For example, let $\mathcal{S}$ be the semiring of non-negative integers, and let $A$ and $B$ be the $n \times n$ matrices with $A_{1,1}=2, B_{1,1}=1$ and all other entries equal to 0 ; then $A \leq_{\mathcal{R}} B$ and $\operatorname{rk}(A)=\operatorname{rk}(B)$ but $A \mathcal{R} B$ since 2 is not invertible in $\mathcal{S}$. 


\section{Bijective linear transformations preserving Green's RELATIONS}

Let $E_{i, j}$ denote the matrix with 1 in the $(i, j)$ th position and 0 elsewhere. It is clear that $M_{n}(\mathcal{S})$ is a free $\mathcal{S}$-module of rank $n^{2}$ having the elements $E_{i, j}$ as basis. Thus any $\mathcal{S}$-linear transformation $T: M_{n}(\mathcal{S}) \rightarrow M_{n}(\mathcal{S})$ is completely determined by the images $T\left(E_{i, j}\right)$.

From now on we assume that $\mathcal{S}$ is a semifield which is not a field (such as for example the tropical or boolean semifield). In this case, it was shown in [3] that the bijective $\mathcal{S}$-linear maps $T$ have a very restricted form. We write $[n]$ for the set $\{1, \ldots, n\}$.

Lemma 3.1. [3, Theorem 2.14]. Let $\mathcal{S}$ be a semifield which is not a field and let $T: M_{n}(\mathcal{S}) \rightarrow M_{n}(\mathcal{S})$ be an $\mathcal{S}$-linear transformation. The following are equivalent

(i) $T$ is bijective;

(ii) $T$ is surjective;

(iii) There exists a permutation $\sigma \in \operatorname{Sym}([n] \times[n])$ and non-zero elements $\alpha_{i, j} \in \mathcal{S}$ such that $T\left(E_{i, j}\right)=\alpha_{i, j} E_{\sigma(i, j)}$ for all $i$ and $j$.

The following fact belongs to folklore; its proof for distributive lattices and some other algebraic structures can be found in [16] and [8]. It is formulated without a proof in [13, Lemma 1] for arbitrary anti-negative semirings. For completeness, we provide here a reduction from Lemma 3.1 in the case of anti-negative semifields, that is, those semifields which are not fields.

Corollary 3.2. Let $\mathcal{S}$ be a semifield which is not a field. The invertible elements of the monoid $M_{n}(\mathcal{S})$ are precisely the monomial matrices, that is, those matrices containing exactly one non-zero element in each row and in each column.

Proof. Since every non-zero element of $\mathcal{S}$ is invertible it is clear that the monomial matrices are invertible; each can be written as a product of an invertible diagonal matrix and a permutation matrix. On the other hand, left multiplication by an invertible matrix $A$ yields a bijective $\mathcal{S}$-linear transformation on $M_{n}(\mathcal{S})$, and so by Lemma 3.1 there exist non-zero elements $\alpha_{i, j} \in$ $\mathcal{S}$ and a permutation $\sigma \in \operatorname{Sym}([n] \times[n])$ such that $A \cdot E_{i, j}=\alpha_{i, j} E_{\sigma(i, j)}$ for all $i, j \in[n]$. Since $A=A \cdot\left(E_{1,1}+\cdots+E_{n, n}\right)=\alpha_{1,1} E_{\sigma(1,1)}+\cdots+\alpha_{n, n} E_{\sigma(n, n)}$, we deduce that $A$ must contain precisely $n$ non-zero entries. It now follows from the fact that $A$ is invertible (and hence cannot contain a zero row) that there must be exactly one non-zero entry in each row. An entirely similar argument, considering right multiplication by $A$, shows that $A$ must be column monomial.

Lemma 3.3. Let $\mathcal{S}$ be a semifield which is not a field and let $T: M_{n}(\mathcal{S}) \rightarrow$ $M_{n}(\mathcal{S})$ be a bijective $\mathcal{S}$-linear transformation. If $T$ preserves any one of $\mathcal{L}, \mathcal{R}, \leq_{\mathcal{L}}$ or $\leq_{\mathcal{R}}$, then there exist $\rho, \chi \in \operatorname{Sym}([n])$ and non-zero elements $x_{i}, y_{j} \in S$ such that

$$
T\left(E_{i, j}\right)=x_{i} y_{j} E_{\rho(i), \chi(j)} \text { for all } i, j \in[n] .
$$


Proof. We prove the result for $\mathcal{L}$; the claim for $\leq_{\mathcal{L}}$ follows since a map preserving $\leq_{\mathcal{L}}$ must clearly preserve $\mathcal{L}$, while the statements for $\mathcal{R}$ and $\leq_{\mathcal{R}}$ are dual.

By Lemma 3.1 there exist elements $\alpha_{i, j} \neq 0 \in \mathcal{S}$ for $i, j \in[n]$ and $\sigma \in$ $\operatorname{Sym}([n] \times[n])$ such that $T\left(E_{i, j}\right)=\alpha_{i, j} E_{\sigma(i, j)}$ for all $i, j \in[n]$. It is an easy consequence of Theorem 2.5 that for any two non-zero elements $a, b \in \mathcal{S}$ and any $i, j, k, l \in[n]$ we have $\left(a E_{i, j}\right) \mathcal{L}\left(b E_{k, l}\right)$ if and only if $j=l$. Thus if $T$ preserves $\mathcal{L}$ then there exists $\chi \in \operatorname{Sym}([n])$ such that

$$
\{\sigma(1, j), \ldots, \sigma(n, j)\}=\{(1, \chi(j)), \ldots,(n, \chi(j))\}
$$

for each $j$. (Informally, this means that $T$ maps 'columns to columns', in the sense that the images of any two matrix units $E_{i, j}$ and $E_{k, j}$ must have their non-zero entries in columns with the same index.)

We claim that there exists $\rho \in \operatorname{Sym}([n])$ such that $\sigma(i, j)=(\rho(i), \chi(j))$ for all $i$ and $j$ (so that $T$ also maps 'rows to rows'). Suppose not. Then there exist $i, j, j^{\prime} \in[n]$ with $j \neq j^{\prime}$ such that $\sigma(i, j)=(m, \chi(j))$ and $\sigma\left(i, j^{\prime}\right)=\left(m^{\prime}, \chi\left(j^{\prime}\right)\right)$ for some $m \neq m^{\prime}$. (In other words, the images of $E_{i, j}$ and $E_{i, j^{\prime}}$ have their non-zero entries in different rows.) To derive the desired contradiction, we now consider separately the cases where $|\mathcal{S}| \geq 3$ and where $\mathcal{S}=\mathbb{B}$ is the boolean semiring (see Remark 2.2 above).

Suppose first that $|\mathcal{S}| \geq 3$. By Remark 2.3 we may choose $\lambda \in \mathcal{S}$ such that $\lambda \neq 0,1$ and $\lambda^{2} \neq 1$. Consider

$$
\begin{aligned}
& A=T^{-1}\left(E_{m, \chi(j)}+E_{m^{\prime}, \chi(j)}+E_{m, \chi\left(j^{\prime}\right)}+E_{m^{\prime}, \chi\left(j^{\prime}\right)}\right), \\
& B=T^{-1}\left(\lambda E_{m, \chi(j)}+E_{m^{\prime}, \chi(j)}+E_{m, \chi\left(j^{\prime}\right)}+\lambda E_{m^{\prime}, \chi\left(j^{\prime}\right)}\right) .
\end{aligned}
$$

Since $m \neq m^{\prime}, \chi(j) \neq \chi\left(j^{\prime}\right)$ and $\lambda^{2} \neq 1$, it is clear that $\operatorname{rk}(T(A))=1$ and $\operatorname{rk}(T(B))=2$. Since factor rank is an $\mathcal{L}$-class invariant we see that $T(A) \not \mathcal{L} T(B)$. On the other hand, it follows easily from the definitions above that there exist non-zero $a, b, c, d \in \mathcal{S}$ and $k, l \neq i$ such that

$$
A=a E_{i, j}+b E_{k, j}+c E_{l, j^{\prime}}+d E_{i, j^{\prime}}, \quad B=\lambda a E_{i, j}+b E_{k, j}+c E_{l, j^{\prime}}+\lambda d E_{i, j^{\prime}} .
$$

Now using the fact that $\lambda$ is invertible in $\mathcal{S}$, we see that $A$ and $B$ have the same row space, and so by Theorem 2.5 we have $A \mathcal{L} B$, providing the required contradiction.

Now consider the case where $\mathcal{S}=\mathbb{B}$. Let $A=E_{i, j}+E_{i, j^{\prime}}$ and $B$ be the matrix with 1 in every entry in the $j$ th and $j^{\prime}$ th columns and 0 elsewhere. Then $A$ and $B$ have the same row space and so by Theorem 2.5 are $\mathcal{L}$-related. However, it is easy to see that the row space of $T(A)$ does not coincide with

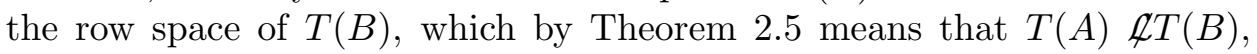
again giving a contradiction.

It remains to show that we can find elements $x_{i}, y_{j} \in \mathcal{S}$ for $i, j \in[n]$ with $\alpha_{i, j}=x_{i} y_{j}$. (Notice that since each $\alpha_{i, j}$ is non-zero this will also imply that the $x_{i}$ 's and $y_{j}$ 's are non-zero.) If we let $R$ be the matrix with $R_{i, j}=\alpha_{i, j}$, what we wish to show is that $R$ has factor rank 1 . Note that for arbitrary permutation matrices $P, Q \in M_{n}(\mathcal{S})$ it is easy to see that $R$ has factor rank 1 precisely if $P R Q$ has factor rank 1.

Let $C$ be the matrix whose entries are all 1 and $D$ the matrix whose first row entries are all 1 and other entries all 0 . Then $C$ and $D$ clearly have the same row space and so by Theorem 2.5 are $\mathcal{L}$-related. Since $T$ preserves $\mathcal{L}$, 
this means $T(C) \mathcal{L} T(D)$, and hence by Theorem 2.5 again, $T(C)$ and $T(D)$ have the same row space. However, from the defining properties of the $\alpha_{i, j}$ it is easy to see that $T(D)$ has only one non-zero row, which is equal to the $\rho(1)$ th row of $T(C)$. Moreover, $T(C)=P R Q$ for permutation matrices $P, Q \in M_{n}(\mathcal{S})$ corresponding to the permutations $\rho$ and $\chi$ respectively. Thus, every row of $T(C)$ must be a multiple of the $\rho(1)$ th row of $T(C)$, showing that $T(C)$, and hence $R$, has factor rank 1 , as required.

Lemma 3.4. Let $\mathcal{S}$ be a semifield which is not a field and let $T: M_{n}(\mathcal{S}) \rightarrow$ $M_{n}(\mathcal{S})$ be a bijective $\mathcal{S}$-linear transformation. If $T$ preserves any of the relations $\mathcal{D}, \mathcal{J}$ or $\leq \mathcal{J}$ then there exist $\rho, \chi \in \operatorname{Sym}([n])$ and non-zero elements $x_{i}, y_{j} \in \mathcal{S}$ for $i, j \in[n]$ such that either

$$
\begin{gathered}
T\left(E_{i, j}\right)=x_{i} y_{j} E_{\rho(i), \chi(j)} \text { for all } i, j \in[n] ; \text { or } \\
T\left(E_{i, j}\right)=x_{i} y_{j} E_{\chi(j), \rho(i)} \text { for all } i, j \in[n] .
\end{gathered}
$$

Proof. Suppose $T$ preserves one of the given relations. By Lemma 3.1 there exist non-zero $\alpha_{i, j} \in \mathcal{S}$ for $i, j \in[n]$ and $\sigma \in \operatorname{Sym}([n] \times[n])$ such that $T\left(E_{i, j}\right)=\alpha_{i, j} E_{\sigma(i, j)}$ for all $i, j \in[n]$.

We claim that for any $i, j, j^{\prime} \in[n]$, the matrices $T\left(E_{i, j}\right)$ and $T\left(E_{i, j^{\prime}}\right)$ have their non-zero entries in either the same row or the same column. Indeed, consider the matrices $E_{i, j}$ and $E_{i, j}+E_{i, j^{\prime}}$. These clearly have the same column space, so by Theorem 2.5 we have $\left(E_{i, j}+E_{i, j^{\prime}}\right) \mathcal{R} E_{i, j}$ and hence also $\left(E_{i, j}+E_{i, j^{\prime}}\right) \mathcal{D} E_{i, j},\left(E_{i, j}+E_{i, j^{\prime}}\right) \mathcal{J} E_{i, j}$ and $\left(E_{i, j}+E_{i, j^{\prime}}\right) \leq \mathcal{J} E_{i, j}$. Since $T$ preserves one of the latter three relations, we have one of $T\left(E_{i, j}+\right.$ $\left.E_{i, j^{\prime}}\right) \mathcal{D} T\left(E_{i, j}\right), T\left(E_{i, j}+E_{i, j^{\prime}}\right) \mathcal{J} T\left(E_{i, j}\right)$ and $T\left(E_{i, j}+E_{i, j^{\prime}}\right) \leq_{\mathcal{J}} T\left(E_{i, j}\right)$, so we must have $T\left(E_{i, j}+E_{i, j^{\prime}}\right) \leq_{\mathcal{J}} T\left(E_{i, j}\right)$ since this is the weakest of the three possibilities. Since factor rank respects the $\mathcal{J}$-order (Remark 2.7) we note that the factor rank of $T\left(E_{i, j}+E_{i, j^{\prime}}\right)$ cannot exceed the factor rank of $T\left(E_{i, j}\right)$, which is clearly seen to be 1 . The only way this can happen is if the two non-zero entries of $T\left(E_{i, j}+E_{i, j^{\prime}}\right)=T\left(E_{i, j}\right)+T\left(E_{i, j^{\prime}}\right)$ lie in either the same row or the same column.

A similar argument (using $\mathcal{L}$ instead of $\mathcal{R}$ ) establishes a dual claim that for any $i, i^{\prime}, j \in[n], T\left(E_{i, j}\right)$ and $T\left(E_{i^{\prime} j}\right)$ have their non-zero entries in either the same row or the same column.

Consider the sets of matrices of the form $\left\{E_{i, k} \mid k \in[n]\right\}$ (which we will call the $i t h$ row set) and of the form $\left\{E_{k, j} \mid k \in[n]\right\}$ (which we will call the $j$ th column set). A simple inductive argument using the two dual claims above shows that $T$ must map every column or row set to either a column or a row set. In fact, we claim that $T$ must either

- map row sets to row sets and column sets to column sets (which we call the standard case); or

- map row sets to column sets and columns sets to row sets (which we call the transpose case).

Indeed, if it did neither of these things, then it would have to map one row set to a row set and another row set to a column set; since the different row sets are disjoint but every row set intersects every column set, this would contradict the fact that $T$ is a bijection.

In the standard case, we define $\rho$ and $\chi$ to be such that $T$ maps the $i$ th row set to the $\rho(i)$ th row set and the $j$ th column set to the $\chi(j)$ th column set. 
Since $T$ is a bijection, they are permutations of $n$, and it is easy to see that $\sigma(i, j)=(\rho(i), \chi(j))$. In the transpose case we define them so that $T$ maps the $i$ th row set to the $\rho(i)$ th column set and the $j$ th column set to the $\chi(j)$ th row set; again they are permutations and this time $\sigma(i, j)=(\chi(j), \rho(i))$.

It remains to show that we can find elements $x_{i}$ and $y_{i}$ with $\alpha_{i, j}=x_{i} y_{j}$. In the standard case, we define $R, C$ and $D$ exactly as in the proof of Lemma 3.3. The same argument as used there shows that $C \mathcal{L} D$ and hence $C \mathcal{D} D, C \mathcal{J} D$ and $C \leq_{\mathcal{J}} D$. By assumption $T$ preserves at least one of the latter three relations, so we have at least one of $T(C) \mathcal{D} T(D), T(C) \mathcal{J} T(D)$ and $T(C) \leq_{\mathcal{J}} T(D)$, which means we must have $T(C) \leq_{\mathcal{J}} T(D)$ since this is the weakest of the three possibilities. Now $T(D)$ has exactly one non-zero row, so must have factor rank 1 , while $T(C)=P R Q$, for some permutation matrices $P, Q \in M_{n}(\mathcal{S})$. Since factor rank respects the $\mathcal{J}$-order (Remark 2.7) it follows that $T(C)$, and hence $R$, has factor rank at most one 1 , which is exactly what we required.

The transpose case is treated by a very similar argument. In particular, $D$ is taken this time to be the matrix with 1 in the first column, we deduce that $C \mathcal{R} D$, that $T(C) \leq_{\mathcal{J}} T(D)$ and that $T(D)$ has exactly one non-zero row, and hence again that $T(C)=(P R Q)^{T}$ has factor rank at most 1.

Theorem 3.5. Let $\mathcal{S}$ be a semifield which is not a field and $T: M_{n}(\mathcal{S}) \rightarrow$ $M_{n}(\mathcal{S})$ a bijective $\mathcal{S}$-linear transformation. Then the following are equivalent:

(i) $T$ preserves $\mathcal{R}$;

(ii) $T$ preserves $\mathcal{L}$;

(iii) $T$ preserves $\leq_{\mathcal{R}}$;

(iv) $T$ preserves $\leq_{\mathcal{L}}$;

(v) there exist invertible (monomial) matrices $P, Q \in M_{n}(\mathcal{S})$ such that for all $X \in M_{n}(\mathcal{S})$, we have $T(X)=P X Q$.

Proof. It is straightforward to verify (directly or using Theorem 2.5) that (v) implies the other four conditions.

Conversely, suppose that one of (i), (ii), (iii) or (iv) holds. Let $\rho, \chi, x_{i}$ and $y_{i}$ be as given by Lemma 3.3. Let $P$ and $Q$ be the monomial matrices given by $P_{\rho(i), i}=x_{i}$ and $Q_{j, \chi(j)}=y_{j}$. The map $M_{n}(\mathcal{S}) \rightarrow M_{n}(\mathcal{S}), X \mapsto P X Q$ is clearly $\mathcal{S}$-linear, so it suffices to show that it agrees with $T$ on the basis elements $E_{i, j}$. For each such, from the definitions of $P$ and $Q$,

$$
\left(P E_{i, j} Q\right)_{r s}= \begin{cases}x_{i} y_{j} & \text { if } r=\rho(i) \text { and } s=\chi(j) \\ 0 & \text { otherwise. }\end{cases}
$$

so that $P E_{i, j} Q=x_{i} y_{j} E_{\rho(i), \chi(j)}=T\left(E_{i, j}\right)$ by Lemma 3.3.

Theorem 3.6. Let $\mathcal{S}$ be a semifield which is not a field and $T: M_{n}(\mathcal{S}) \rightarrow$ $M_{n}(\mathcal{S})$ a bijective $\mathcal{S}$-linear transformation. Then the following are equivalent:

(i) $T$ preserves $\mathcal{D}$;

(ii) $T$ preserves $\mathcal{J}$;

(iii) $T$ preserves $\leq \mathcal{J}$; 
(iv) there exist invertible (monomial) matrices $P, Q \in M_{n}(\mathcal{S})$ such that either $T(X)=P X Q$ for all $X \in M_{n}(\mathcal{S})$, or $T(X)=P X^{T} Q$ for all $X \in M_{n}(\mathcal{S})$.

Proof. Again, it is straightforward to verify (directly or using Theorem 2.5) that (iv) implies the other three conditions. For the converse, suppose one of (i), (ii) or (iii) holds and let $\rho, \chi, x_{i}$ and $y_{i}$ be given this time by Lemma 3.4. In the standard case (in the terminology of the proof of Lemma 3.4) define $P$ and $Q$ as in the proof of Theorem 3.5 and we have $T(X)=P X Q$ for all $X$ by exactly the same argument but using Lemma 3.4 in place of Lemma 3.3 at the end.

In the transpose case, define $P$ and $Q$ to be the monomial matrices with $P_{\chi(j), j}=y_{j}$ and $Q_{i, \rho(i)}=x_{i}$. The map $X \mapsto P X^{T} Q$ is easily seen to be $\mathcal{S}$-linear, and a simple calculation gives

$$
\left(P\left(E_{i, j}\right)^{T} Q\right)_{r s}= \begin{cases}y_{j} x_{i} & \text { if } r=\chi(j) \text { and } s=\rho(i) \\ 0 & \text { otherwise. }\end{cases}
$$

so that $P\left(E_{i, j}\right)^{T} Q=x_{i} y_{j} E_{\chi(j), \rho(i)}=T\left(E_{i, j}\right)$ by Lemma 3.4 again.

\section{THE $\mathcal{H}$ RELATION}

So far we have proved relatively little about the $\mathcal{H}$ relation. We begin with a lemma analogous to, but weaker than, Lemmas 3.3 and 3.4 above.

Lemma 4.1. Let $\mathcal{S}$ be a semifield which is not a field and let $T: M_{n}(\mathcal{S}) \rightarrow$ $M_{n}(\mathcal{S})$ be a bijective $\mathcal{S}$-linear transformation. If $T$ preserves $\mathcal{H}$ or $\leq_{\mathcal{H}}$, then there exist $\rho, \chi \in \operatorname{Sym}([n])$ and non-zero elements $\alpha_{i, j} \in \mathcal{S}$ such that either

$$
\begin{gathered}
T\left(E_{i, j}\right)=\alpha_{i, j} E_{\rho(i), \chi(j)} \text { for all } i, j \in[n] ; \text { or } \\
T\left(E_{i, j}\right)=\alpha_{i, j} E_{\chi(j), \rho(i)} \text { for all } i, j \in[n] .
\end{gathered}
$$

Proof. We prove the result for $\mathcal{H}$; the claim for $\leq_{\mathcal{H}}$ follows since a map preserving $\leq_{\mathcal{H}}$ must clearly preserve $\mathcal{H}$. Let $T: M_{n}(\mathcal{S}) \rightarrow M_{n}(\mathcal{S})$ be a bijective $\mathcal{S}$-linear transformation preserving $\mathcal{H}$. By Lemma 3.1 there exist $\alpha_{i, j} \in \mathcal{S}$ and $\sigma \in \operatorname{Sym}([n] \times[n])$ such that $T\left(E_{i, j}\right)=\alpha_{i, j} E_{\sigma(i, j)}$ for all $i, j \in[n]$. Define row sets and column sets of basic matrices as in the proof of Lemma 3.4. Just as there, we need to show that $T$ maps row sets and column sets to row sets and column sets.

Suppose not. Then there must exist $i, j, k, l \in[n]$ with $i \neq j$ and $k \neq l$ such that either

- $\sigma(i, k)=(m, q)$ and $\sigma(j, l)=(p, q)$; or

- $\sigma(i, k)=(q, m)$ and $\sigma(j, l)=(q, p)$,

for some $m, p, q$ with $m \neq p$. We consider the first case, the second being dual.

Consider the matrices $A=E_{i, k}+E_{j, l}$ and $B=E_{i, l}+E_{j, k}$. It is easy to see (for example by Theorem 2.5) that $A \mathcal{H} B$. Now $T(A)$ has non-zero entries only in the $(m, q)$ and $(p, q)$ positions, and it is also easy to show that any matrix $\mathcal{H}$-related to $T(A)$ must have non-zero entries in these same two positions. Since $T$ preserves the $\mathcal{H}$ relation we have $T(A) \mathcal{H} T(B)$, so $T(B)$ must have this form, thus we must have $\{\sigma(i, l), \sigma(j, k)\}=\{(m, q),(p, q)\}=$ 
$\{\sigma(i, k), \sigma(j, l)\}$ which clearly contradicts the fact that $\sigma$ is a permutation of $[n] \times[n]$.

This establishes that $T$ maps row sets and column sets to row sets and column sets. We now reason exactly as in the proof of Lemma 3.4 to deduce that it either maps row sets to row sets and column sets to column sets (the standard case), or row sets to column sets and column sets to row sets (the transpose case). The construction of the permutations $\chi$ and $\rho$ is then also just as in the proof of Lemma 3.4.

We thank the anonymous referee for suggesting the proof of the following lemma, which allows us to strengthen what was a partial result in the first draft of this article.

Lemma 4.2. Let $\mathcal{S}$ be a semifield which is not a field and let $T: M_{2}(\mathcal{S}) \rightarrow$ $M_{2}(\mathcal{S})$ be the linear bijection given by $T(X)=M \circ X$ where $M$ is a fixed $2 \times 2$ matrix whose entries are all non-zero elements of $\mathcal{S}$, and $\circ$ denotes the entry-wise Hadamard product. If $T$ preserves $\mathcal{H}$, then $M$ has rank 1.

Proof. Let $M=\left(\begin{array}{ll}a & b \\ c & d\end{array}\right)$ where $a, b, c, d$ are non-zero elements of $\mathcal{S}$ and suppose that $T$ is as given and preserves $\mathcal{H}$. For each fixed non-zero $k \in \mathcal{S}$ it is easy to see that the matrices

$$
U_{k}=\left(\begin{array}{cc}
k & 1 \\
1 & k
\end{array}\right) \text { and } V_{k}=\left(\begin{array}{cc}
1 & k \\
k & 1
\end{array}\right)
$$

are $\mathcal{H}$-related. Since $T$ preserves $\mathcal{H}$ it then follows that for each non-zero $k$ we also have $T\left(U_{k}\right) \mathcal{H} T\left(V_{k}\right)$ and $T\left(T\left(U_{k}\right)\right) \mathcal{H} T\left(T\left(V_{k}\right)\right)$. Taking $k=a^{-1} b c d^{-1}$ we find that

$$
T\left(T\left(U_{k}\right)\right)=\left(\begin{array}{cc}
k a^{2} & b^{2} \\
c^{2} & k d^{2}
\end{array}\right)=\left(\begin{array}{l}
a b \\
c d
\end{array}\right)\left(\begin{array}{ll}
c d^{-1} & a^{-1} b
\end{array}\right)
$$

is a rank 1 matrix, and since rank is an invariant of $\mathcal{H}$-classes we see that

$$
T\left(T\left(V_{k}\right)\right)=\left(\begin{array}{cc}
a^{2} & b^{2} k \\
c^{2} k & d^{2}
\end{array}\right)
$$

must also have rank 1. Remark 2.6 then gives that for this value of $k$ we have $k^{2} a^{2} d^{2}=b^{2} c^{2}$ and $a^{2} d^{2}=k^{2} b^{2} c^{2}$, from which we may deduce that $k^{4}=1$. Remark 2.3 then yields that $k=1$, whereupon (by our choice of $k$ ) we find that $a d=b c$. By Remark 2.6 again, $M$ has rank 1 .

This argument can be extended, allowing us to prove:

Theorem 4.3. Let $\mathcal{S}$ be a semifield which is not a field and let $T: M_{n}(\mathcal{S}) \rightarrow$ $M_{n}(\mathcal{S})$ be a bijective $\mathcal{S}$-linear transformation. If $T$ preserves $\mathcal{H}$ or $\leq_{\mathcal{H}}$, then there exist $\rho, \chi \in \operatorname{Sym}([n])$ and non-zero elements $x_{i}, y_{j} \in \mathcal{S}$ for $i, j \in[n]$ such that either

$$
\begin{aligned}
& T\left(E_{i, j}\right)=x_{i} y_{j} E_{\rho(i), \chi(j)} \text { for all } i, j \in[n] ; \text { or } \\
& T\left(E_{i, j}\right)=x_{i} y_{j} E_{\chi(j), \rho(i)} \text { for all } i, j \in[n] .
\end{aligned}
$$

Proof. We prove the result for $\mathcal{H}$, again noting that the claim for $\leq_{\mathcal{H}}$ follows from this. By Lemma 4.1 there exist permutation matrices $P, Q \in M_{n}(\mathcal{S})$ and a matrix $A \in M_{n}(\mathcal{S})$ whose entries are all non-zero such that either 
$T(X)=P(A \circ X) Q$ for all $X \in M_{n}(\mathcal{S})$, or $T(X)=P(A \circ X)^{T} Q$ for all $X \in M_{n}(\mathcal{S})$, where $\circ$ denotes the Hadamard product. It therefore suffices to show that the matrix $A=\left(\alpha_{i, j}\right)$ has factor rank 1 . Suppose not for contradiction. Then $A$ must contain a $2 \times 2$ submatrix

$$
M=\left(\begin{array}{cc}
\alpha_{l, p} & \alpha_{l, q} \\
\alpha_{m, p} & \alpha_{m, q}
\end{array}\right)
$$

of factor rank 2. Consider the map $T^{\prime}: M_{n}(\mathcal{S}) \rightarrow M_{n}(\mathcal{S})$ given by $T^{\prime}(X)=$ $A \circ X$. Since $T$ is a linear bijection preserving $\mathcal{H}$, so too is $T^{\prime}$.

Let $k$ be an arbitrary non-zero element of $\mathcal{S}$ and consider the matrices

$$
\begin{aligned}
& U=k E_{l, p}+E_{l, q}+E_{m, p}+k E_{m, q}, \\
& V=E_{l, p}+k E_{l, q}+k E_{m, p}+E_{m, q} .
\end{aligned}
$$

Up to reordering, $U$ and $V$ have the same rows and also the same columns. Thus $U \mathcal{H} V$. Now let $U^{\prime}=T^{\prime}\left(T^{\prime}(U)\right)$ and $V^{\prime}=T^{\prime}\left(T^{\prime}\left(U^{\prime}\right)\right)$. Since $T^{\prime}$ preserves $\mathcal{H}$, we find that $U^{\prime} \mathcal{H} V^{\prime}$, where

$$
\begin{aligned}
U^{\prime} & =k \alpha_{l, p}^{2} E_{l, p}+\alpha_{l, q}^{2} E_{l, q}+\alpha_{m, p}^{2} E_{m, p}+k \alpha_{m, q}^{2} E_{m, q}, \\
V^{\prime} & =\alpha_{l, p}^{2} E_{l, p}+k \alpha_{l, q}^{2} E_{l, q}+k \alpha_{m, p}^{2} E_{m, p}+\alpha_{m, q}^{2} E_{m, q} .
\end{aligned}
$$

Taking $k=\alpha_{l, p}^{-1} \alpha_{l, q} \alpha_{m, p} \alpha_{m, q}^{-1}$ in the above, the matrix $U^{\prime}$ has factor rank 1 ; it is the product of the column vector $x$ and the row vector $y$ with nonzero entries given as follows:

$$
x_{l}=\alpha_{l, p} \alpha_{l, q}, \quad x_{m}=\alpha_{m, p} \alpha_{m, q}, \quad y_{l}=\alpha_{m, p} \alpha_{m, q}^{-1}, \quad y_{m}=\alpha_{l, p}^{-1} \alpha_{l, q} .
$$

Since $V^{\prime} \mathcal{H} U^{\prime}$, the matrix $V^{\prime}$ must also have factor rank 1 . Reasoning as in Lemma 4.2 we obtain $k^{4}=1$. Remark 2.3 then gives $k=1$, and so by our choice of $k$ we have $\alpha_{l, q} \alpha_{m, p}=\alpha_{l, p} \alpha_{m, q}$, contradicting that the submatrix $M$ has rank 2 .

Combining the previous result with Theorem 3.6 now gives:

Corollary 4.4. Let $\mathcal{S}$ be a semifield which is not a field and $T: M_{n}(\mathcal{S}) \rightarrow$ $M_{n}(\mathcal{S})$ a bijective $\mathcal{S}$-linear transformation. Then the following are equivalent:

(i) $T$ preserves $\mathcal{D}$;

(ii) $T$ preserves $\mathcal{J}$;

(iii) $T$ preserves $\leq \mathcal{J}$;

(iv) $T$ preserves $\mathcal{H}$;

(v) $T$ preserves $\leq_{\mathcal{H}}$;

(vi) there exist invertible (monomial) matrices $P, Q \in M_{n}(\mathcal{S})$ such that either $T(X)=P X Q$ for all $X \in M_{n}(\mathcal{S})$, or $T(X)=P X^{T} Q$ for all $X \in M_{n}(\mathcal{S})$.

Corollary 4.4 motivates some further natural definitions.

We say that a map $T: M_{n}(\mathcal{S}) \rightarrow M_{n}(\mathcal{S})$ exchanges two binary relations $\tau$ and $\theta$ if $x \tau y \Longrightarrow T(x) \theta T(y)$ and $x \theta y \Longrightarrow T(x) \tau T(y)$. We say that it strongly exchanges $\tau$ with $\theta$ if $x \tau y \Longleftrightarrow T(x) \theta T(y)$ and $x \theta y \Longleftrightarrow$ $T(x) \tau T(y)$. Notice that a map $T: M_{n}(\mathcal{S}) \rightarrow M_{n}(\mathcal{S})$ (strongly) preserves $\mathcal{L}$ and $\mathcal{R}$ if and only if the map $T^{\prime}: X \mapsto T(X)^{T}$ (strongly) exchanges $\mathcal{L}$ with $\mathcal{R}$. 
Corollary 4.5. Let $\mathcal{S}$ be a semifield which is not a field. If a bijective $\mathcal{S}$ linear transformation on $M_{n}(\mathcal{S})$ preserves any of $\mathcal{H}, \mathcal{D}, \mathcal{J}, \leq_{\mathcal{H}}$ and $\leq_{\mathcal{J}}$ then it:

- strongly preserves $\mathcal{H}, \mathcal{D}, \mathcal{J}, \leq_{\mathcal{H}}$ and $\leq_{\mathcal{J}}$; and

- either strongly preserves $\mathcal{L}, \mathcal{R}, \leq_{L}$ and $\leq_{\mathcal{R}}$ or else strongly exchanges $\mathcal{L}$ with $\mathcal{R}$ and strongly exchanges $\leq_{\mathcal{L}}$ with $\leq_{\mathcal{R}}$.

Corollary 4.6. Let $\mathcal{S}$ be a semifield which is not a field and $T: M_{n}(\mathcal{S}) \rightarrow$ $M_{n}(\mathcal{S})$ a bijective $\mathcal{S}$-linear transformation. Then the following are equivalent:

(i) $T$ exchanges $\mathcal{L}$ with $\mathcal{R}$;

(ii) $T$ exchanges $\leq_{\mathcal{L}}$ with $\leq_{\mathcal{R}}$;

(iii) $T$ strongly exchanges $\mathcal{L}$ with $\mathcal{R}$;

(iv) $T$ strongly exchanges $\leq_{\mathcal{L}}$ with $\leq_{\mathcal{R}}$;

(v) there exist invertible (monomial) matrices $P, Q \in M_{n}(\mathcal{S})$ such that for all $X \in M_{n}(\mathcal{S})$, we have $T(X)=P X^{T} Q$.

Corollary 4.7. Let $\mathcal{S}$ be a semifield which is not a field. If a bijective $\mathcal{S}$ linear transformation on $M_{n}(\mathcal{S})$ preserves any of $\mathcal{L}, \mathcal{R}, \leq_{\mathcal{L}}$ and $\leq_{R}$ then it strongly preserves $\mathcal{L}, \mathcal{R}, \mathcal{H}, \mathcal{D}, \mathcal{J}, \mathcal{H}, \leq_{\mathcal{H}}, \leq_{\mathcal{L}}, \leq_{\mathcal{R}}$ and $\leq_{J}$.

\section{ACKNOWLEDGMENTS}

The research contained in this article was started in 2012 during a visit of the first author to the University of Manchester, funded by EPSRC grant EP/I005293/1 (Nonlinear Eigenvalue Problems: Theory and Numerics). He is grateful to the School of Mathematics and the Tropical Mathematics Group for their warm hospitality. He also thanks RFBR grant 15-01-01132 for partial financial support of his research. The authors thank the referee for his/her valuable suggestions, and in particular, for suggesting the proof of Lemma 4.2.

\section{REFERENCES}

[1] M. Akian, R. Bapat, and S. Gaubert. Max-plus algebra. Handbook of Linear Algebra, V. 39. Chapman and Hall, 2006.

[2] M. Akian, S. Gaubert, and A. Guterman, Linear independence over tropical semirings and beyond, Contemporary Mathematics, AMS, 495 (2009) 1-38.

[3] L. Beasley and A. Guterman, Linear preservers of extremes of rank inequalities over semirings: the factor rank, J. of Math. Sciences (New York). 131 no. 5 (2005) 59195938 .

[4] J. Dieudonné, Sur une généralisation du groupe orthogonal à quatre variables. Arch. Math. 1 (1949) 282-287.

[5] G. Frobenius, Über die Darstellung der endlichen Gruppen durch lineare Substitutionen, Sitzungsber, Preuss. Akad. Wiss (Berlin), Berlin, 1897, 994-1015.

[6] A. E. Guterman, Matrix invariants over semirings, Handbook of Algebra ed.: M. Hazewinkel, Elsevier, North-Holland, 6 (2009) 3-33.

[7] C. Hollings and M. Kambites, Tropical matrix duality and Green's $\mathcal{D}$ relation, J. London Math. Soc. 86, no. 2 (2012) 520-538.

[8] S. N. Il'in, Invertibility of matrices over ordered algebraic systems, Sib. Mat. J. 39 no. 3 (1998) 551-559 [in Russian].

[9] M. Johnson and M. Kambites, Green's $J$-order and the rank of tropical matrices, $J$. of Pure Appl. Algebra, 217, no. 2 (2013) 280-292. 
[10] M. Johnson and M. Kambites, Multiplicative structure of $2 \times 2$ tropical matrices, Linear Algebra Appl., 435 (2011) 499-512.

[11] C.-K. Li and N.K. Tsing, Linear preserver problems: A brief introduction and some special techniques, Linear Algebra Appl. 162-164 (1992) 217-235.

[12] S. Pierce et al, A survey of linear preserver problems, Linear and Multilinear Algebra, 33 (1992) 1-119.

[13] O.A. Pshenitsyna, Maps preserving invertibility for matrices over semirings, Uspekhi Mat.Nauk 64 no. 1 (2009) 157-158 [in Russian].

[14] G. Tahar, Ordered algebraic structures and classification of semifields, arXiv:1709.06923v1 [math.AG].

[15] I. Schur, Einige Bemerkungen zur Determinantentheorie. Akad. Wiss. Berlin: S.-Ber. Preuß., (1925) 454-463.

[16] L.A. Skornyakov, Invertibility of matrices over distributive structures, Sib. Mat. J. 27 no. 2 (1986) 182-185 [in Russian].

[17] D. Wilding, M. Johnson and M. Kambites, Exact rings and semirings, J. Algebra, 388 (2013) 324-337. 